Classification

Physics Abstracts

$68.35 \mathrm{Bs}-07.90+\mathrm{c}-87.15 \mathrm{By}-06.50 \mathrm{Dc}$

\title{
A scanning force microscope designed for applied surface studies
}

\author{
Ragnar Erlandsson, Roger Wigren and Lars Olsson \\ Laboratory of Applied Physics, Department of Physics and Measurement Technology, Linköping \\ University, S-581 83 Linköping, Sweden
}

(Received October 13, 1990; accepted January 04, 1991)

\begin{abstract}
Résumé. - Nous présentons un microscope de force à balayage travaillant dans le mode force attractive et utilisant une détection de levier par interférométrie laser. L'instrument est destiné à des applications multiples et les critères de réalisation visent un usage facile et une grande versatilité. Une tête interchangeable permet de grandes fenêtres de balayage sans compromettre les performances obtenues à forts grossissements. L'ensemble de logiciels mis au point contrôle l'acquisition des données et permet un traitement d'image post-acquisition comprenant la manipulation d'images multiples sur l'écran et la restitution tridimensionnelle des images. La résolution latérale courante est meilleure que $30 \AA$ et la verticale meilleure que $1 \AA$. Les performances de l'instrument sont illustrées par des données sur la fibrìnogène absorbée sur de la silice hydrophobe et montre pour la première fois la possibilité d'observation de la morphologie de molécules biologiques individuelles avec un microscope de force travaillant dans le mode attractif.
\end{abstract}

\begin{abstract}
We present a Scanning Force Microscope working in the attractive force mode and using an optical fiber equipped laser interferometer as lever deflection detector. The instrument is built for general purpose use and leading design criteria have been versatility and easy operation. An interchangeable scanner system allows large scan sizes without compromising the performance working with high magnifications. A comprehensive software package has been developed that controls data acquisition and permits an extensive post-acquisition image processing including 3-D rendering and handling of multiple images on the screen. The lateral resolution routinely achieved is better than $30 \AA$ and the height resolution is better than $1 \AA$. The performance of the instrument is exemplified by data on fibrinogen adsorbed on hydrofobic silicon oxide and demonstrates for the first time the ability to observe the morphology of individual biological molecules when operating the SFM in the attractive mode.
\end{abstract}

\section{Introduction.}

The Scanning Force Microscope, SFM, also known as Atomic Force Microscope, was invented in 1986 by Binnig and co. workers [1], and has now evolved into an established technique for surface characterization. The ability to work with both short range repulsive forces, which can give atomic resolution, and longer range attractive forces (van der Waals, electrostatic, magnetic) has added 
to the versatility of the method. Both topographic imaging and mapping of force fields due to magnetic or electrostatic distributions is now done on a routine basis, and the technique has been applied to a whole variety of problems including single crystals [2], biological systems [3], magnetic recording media [4], microelectronics [5], friction [6] etc.

The SFM technique can be used on insulating as well as conducting samples which is not the case for Scanning Tunneling Microscopy. It has the advantages over conventional electron microscopy techniques such as TEM and SEM in that it gives topographic information directly and that sample preparation is virtually eliminated. So far most Force Microscopes have been operated in air at room temperature, but there are also examples of measurements performed in liquids [7] and at low temperatures [8]. During the last year, the first UHV force microscope has appeared $[9,10]$, and this important development will certainly strengthen force microscopy as a technique for basic surface studies.

In this paper we present a Scanning Force Microscope operating in air together with some data on fibrinogen molecules adsorbed on hydrophobic silicon oxide.

\section{Instrument design.}

2.1 DESIGN CONSIDERATIONS. - The ergonomic aspects of the apparatus such as easy sample exchange, good visibility of the sample and automatic tip-approach have been specifically emphasized in order to make the instrument versatile and achieve quick sample turn-around time. We have chosen a modular design that permits quick exchange of the scanner system in order to allow a wide variety of scan ranges without sacrifice in ultimate resolution and stability. A sample orientation close to horizontal was chosen as measurements in the liquid phase are planned. The lever deflection detector is based on a laser interferometer using an optical fiber, a well proven and reliable system that also gives complete freedom in the orientation of the scanner system relative to the optical equipment. The computer software runs on a Macintosh II computer and is designed to perform on-line data acquisition as well as post-acquisition data processing.

2.2 MECHANICAL CONFIGURATION. - The central structural element of the microscope is the base plate that supports the top plate on which the force sensor is mounted, see figure 1 . The scanner module is attached to the base plate and can easily be changed. The top-plate is resting on three hemispherical pivot-points, one of which can be vertically adjusted by a motorized micrometer to provide the coarse approach. This geometry gives good access when observing the sample with a binocular microscope and makes sample change easy as the top plate can be raised to a vertical position, leaving the sample region completely free.

In order to isolate the microscope from building vibrations and acoustical interference, the apparatus is mounted in a Plexiglas box that rests on air bladders. Inside this enclosure, the entire instrument including the optics is mounted on a $30 \times 60 \mathrm{~cm}$ optical breadboard which is suspended from the top of the box using rubber springs giving a vertical resonance frequency of $0.7 \mathrm{~Hz}$. An additional high frequency isolation stage is provided by a stack of five steel plates separated by elastomer spacers that support the base plate.

2.3 FORCE SENSOR. - The force sensor consists of the cantilever/tip unit, mounted on a piezoelectric bimorph, and the laser interferometer lever deflection detector, see figure 2 . The light source is a $1 \mathrm{~mW}$ HeNe laser (Uniphase $1101 \mathrm{P}, \lambda=632.8 \mathrm{~nm}$ ) with plane polarized output and the light is carried to the cantilever through an optical fiber with $4 \mu \mathrm{m}$ core diameter. The deflection signal is derived from the intensity at the photodiode which is determined by the phase between the light reflected from the cantilever and that reflected at the glass-air interface at the fiber end. 


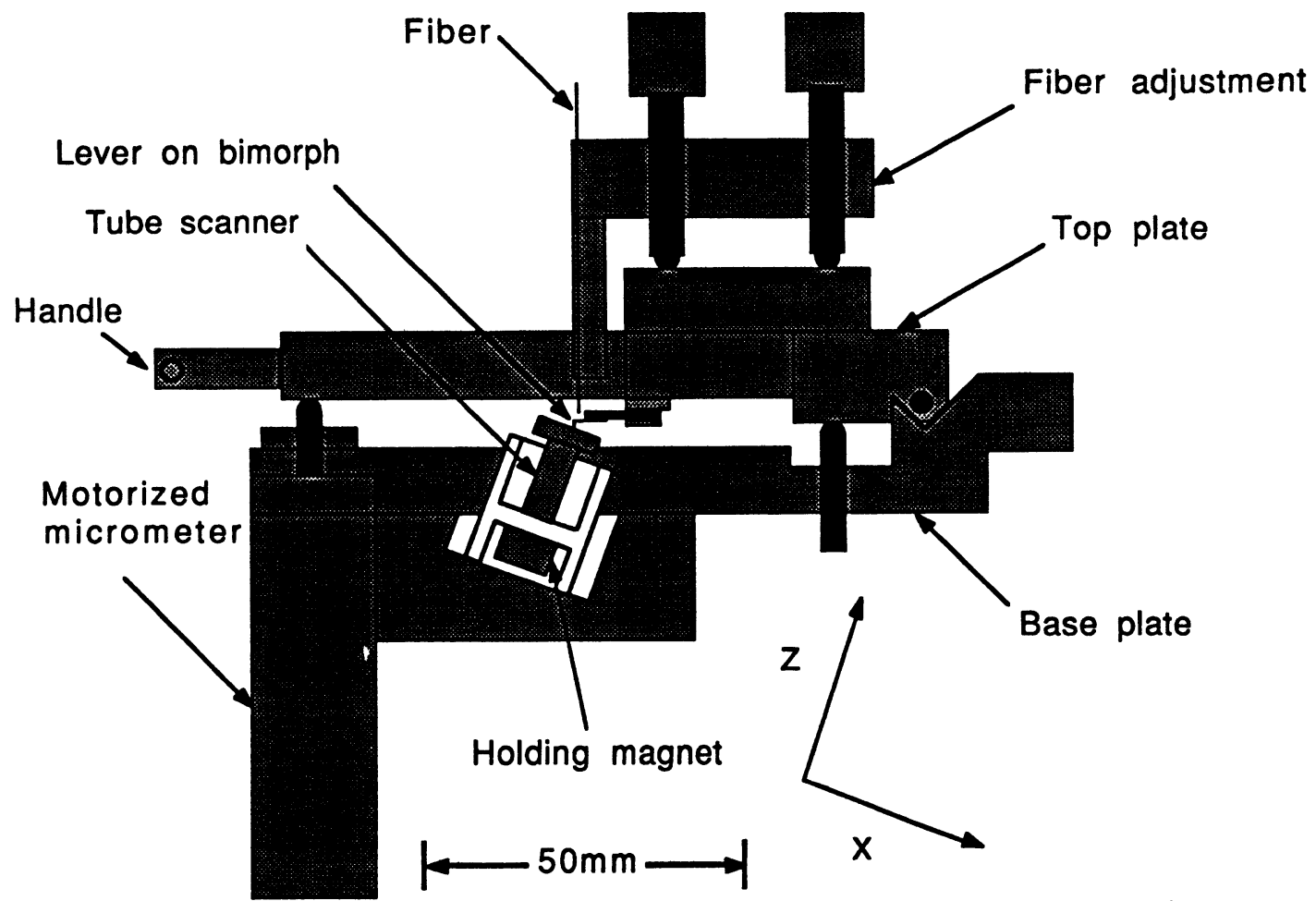

a)

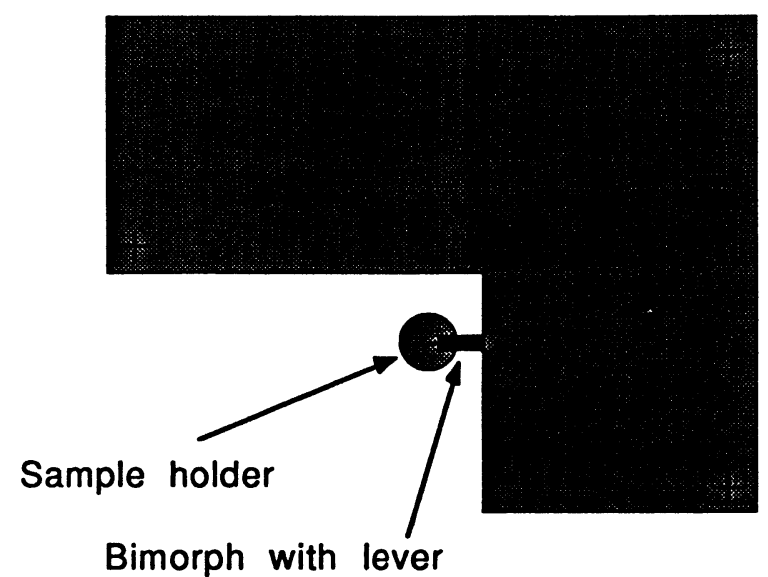

b)

Fig. 1. - (a) Side view of the microscope with a cut through the lower part to make the tube-scanner visible. b) The top-plate seen from above. The crosses indicate the support points.

The small size of this interference cavity, only a few $\mu \mathrm{m}$, is advantageous as it decreases the sensitivity to laser frequency variations [11]. The static position of the cantilever can be adjusted using the piezoelectric bimorph in order to give a phase shift of $\pi / 2$ between the two interfering beams which maximizes the interferometer sensitivity. For small deflections around this position 


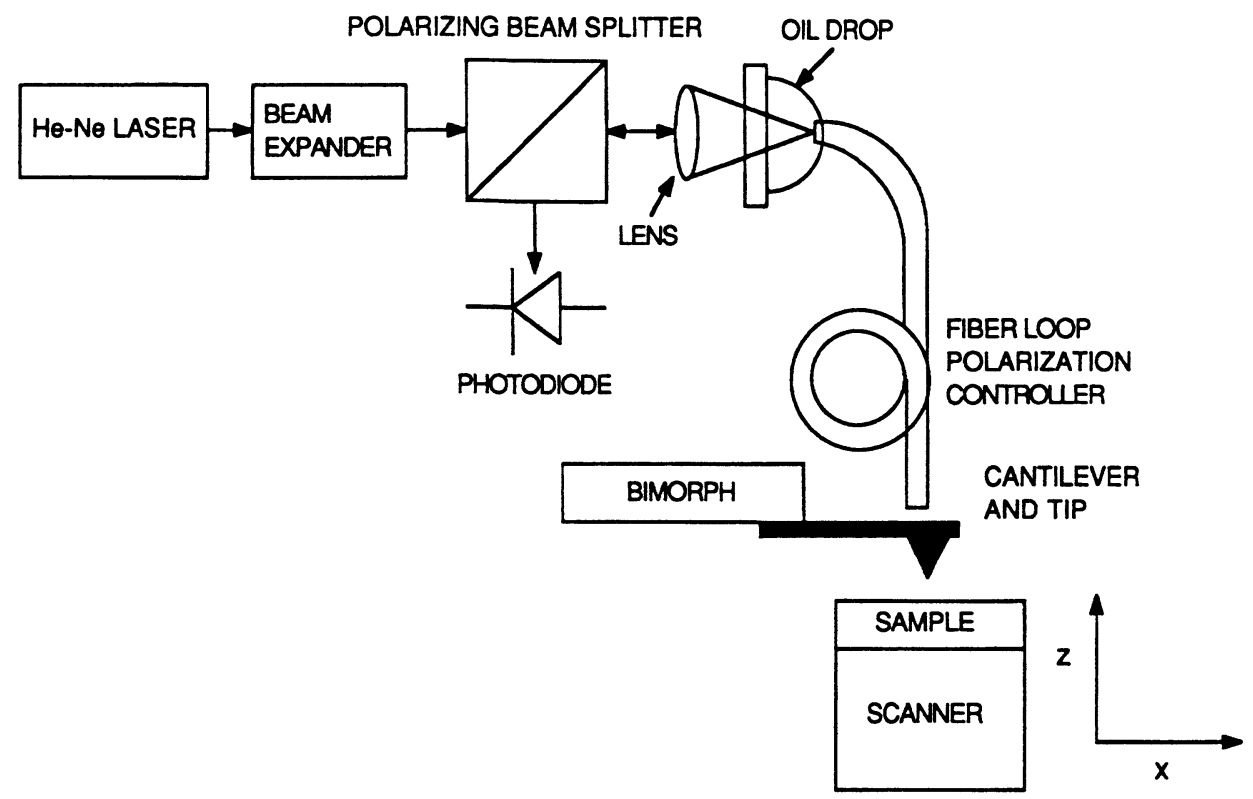

Fig. 2. - Schematic diagram of the force sensor.

the intensity will vary linearly with lever-displacement and the sensitivity can easily be calibrated by moving the lever a distance corresponding to a path difference of half a wavelength.

To avoid reflections when the light enters the fiber, the end is immersed in a small volume of index matching fluid that will keep the reflecting interface out of focus. Stability problems due to retroreflection of light into the laser is minimized using a method that isolates the laser from the reflected beam and that works as follows: the polarization plane of the beam returning through the fiber is rotated $90^{\circ}$ with respect to the beam leaving the laser as a result of the double passage through the fiber loop which acts as a $\lambda / 4$ plate due to stress induced birefringence in the fiber material [12]. The polarizing beam splitter deflects the returning beam into the detector while blocking the path to the laser thus avoiding stabillity problems due to retroreflection into the laser.

Reference [13] gives a detailed analysis of this type of deflection sensor and shows that the noise level above $2 \mathrm{kHz}$ can be reduced to within a factor of 2.5 of the shot noise limit of the detector circuit. Figure 3 shows the frequency distribution of the noise in the detector signal of the instrument described here.

As the deflection sensor described above is suitable for ac as well as dc measurements, the direct force measurement technique [14] as well as the resonance technique [5,15] can be used. In the former case, the lever deflection is directly used to obtain the force, and the feedback electronics regulates the $z$-position of the sample to give constant force during the scan. This technique is normally used in the repulsive force regime and puts stringent requirements on the dc-stability of the deflection sensor. Using the resonance technique, the shift in resonance frequency of the cantilever due to the force interaction is used as control signal, which makes the tip follow contours of constant force derivative, see section 3.1. This technique is predominantly used in the attractive force regime. The instrument described here has so far been operated exclusively in the resonance mode.

The cantilever/tip unit is made from a tungsten wire that is bent and electrochemical etched 


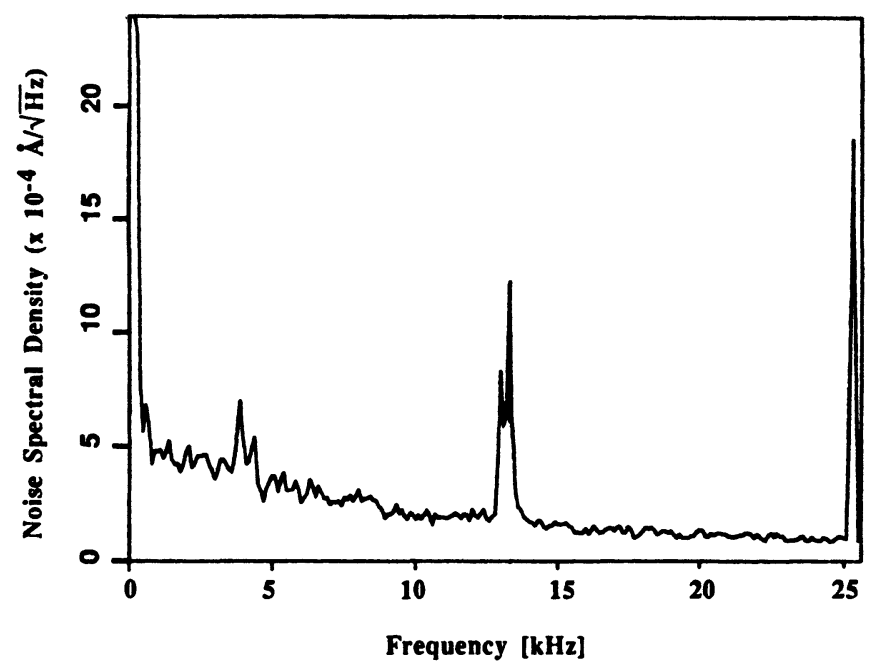

Fig. 3. - Noise spectrum of the interferometer signal with a tungsten cantilever mounted. The double peak at $13.3 \mathrm{kHz}$ is the thermally excited resonance of the cantilever.

using a commercial film developer (Neutol-S) as electrolyte [16]. The $90^{\circ}$ bend between the lever section and the tip section is made before etching. In the first step, the diameter of the $0.1 \mathrm{~mm}$ wire (both tip and lever section) is reduced to $15-25 \mu \mathrm{m}$ by a $7 \mathrm{~V}$ ac etch. In the second step only the tip section is immersed in the electrolyte and the etch is terminated when the immersed part of the wire breaks off. That the tip is formed as a result of a fracture under the stress caused by the weight of the immersed wire is believed to increase the probability of obtaining small tip radii. TEM measurements have shown that tip radii of better then $200 \AA$ can be achieved. The length of the etched lever section is typically $1 \mathrm{~mm}$ and the tip section is $0.1-0.3 \mathrm{~mm}$ long, giving a resonance frequency in the $20 \mathrm{kHz}$ range and a force constant between 10 and $100 \mathrm{~N} / \mathrm{m}$.

2.4 SCANNER SYSTEM. - To avoid the compromise between large scan size, thermal stability and rigidity, we have designed two scanner systems that can easily be interchanged. When a lateral sample displacement less then $5 \mu \mathrm{m}$ is needed, a tube scanner [17] made from the piezoelectric material PZT5A [18] is used. The tube length, diameter and wall thickness are 12.7, 6.3 and $0.5 \mathrm{~mm}$ respectively, giving a maximum lateral range of $5.5 \mu \mathrm{m}$ when applying scan voltages between $+150 \mathrm{~V}$ and $-150 \mathrm{~V}$ differentially to the opposite outer segments of the tube [19]. Motion in the $z$ direction is obtained by applying a ground referenced voltage to the inner electrode, giving a maximum range of $2.1 \mu \mathrm{m}$ when applying voltages between $-150 \mathrm{~V}$ and $+150 \mathrm{~V}$. The maximum lateral scan range used under normal conditions is $2 \mu \mathrm{m}$.

The piezo-tube is mounted in a macor structure which is held by friction against the scanner support block using the force from a magnet (Fig. 1a). By sliding this structure in the $x$ and $y$ directions a coarse adjustement with $7 \mathrm{~mm}$ range is obtained without compromising the rigidity. The whole tube scanner assembly is tilted $20^{\circ}$ with respect to the lever plane to allow a well defined tip contact when using small cantilevers. The sample holder is electrically isolated from the tube scanner, and can be biased with respect to the tip.

For scan ranges up to $30 \mu \mathrm{m}$, an additional scanner system based on three orthogonally connected commercial piezo translators [20] is used. Coarse adjustement is in this case provided by a commercially available $x-y$ table with a $5.5 \mathrm{~mm}$ range on which the translators are mounted. 


\section{Control system.}

3.1 FEEDBACK AND SCAN-DRIVER SYSTEMS. - The general layout of the control system used with the tube scanner is shown in figure 4. The central part is the feedback regulator that adjusts the voltage to the $z$-electrode of the scanner to obtain a constant tip-sample separation. In attractive mode measurements using the resonance method, the cantilever is oscillated (typically a few $\AA$ amplitude) near the resonance frequency by applying an ac signal to the bimorph while measuring the oscillation amplitude with lock-in detection. Provided that no damping of the resonance occurs, the shift of this amplitude signal is related, through the shape of the resonance curve, to the shift of lever resonance frequency due to the force interaction. This frequency shift, $\Delta f$, is related to the force derivative between the tip and the sample, $F^{\prime}$, through the relation

$$
\Delta f=F^{\prime} f_{\text {res }} / 2 k
$$

where $f_{\text {res }}$ is the resonance frequency of the undisturbed lever and $k$ is the force constant. If the feedback regulator adjusts the $z$-voltage to the scanner in order to keep the amplitude shift at a preset value, the tip will follow traces of constant force derivative. The maximum sensitivity is obtained if the oscillator driving the lever vibration is tuned to a frequency corresponding to the inflection point of the resonance curve. For tip-sample separations less than approximately $5 \mathrm{~nm}$, a capillary condensation effect leading to a liquid bridge between tip and sample is normally contributing to the force interaction [2,21]. In this case the amplitude decrease is also an effect of damping, and the simple correlation between force derivative and amplitude no longer exists.

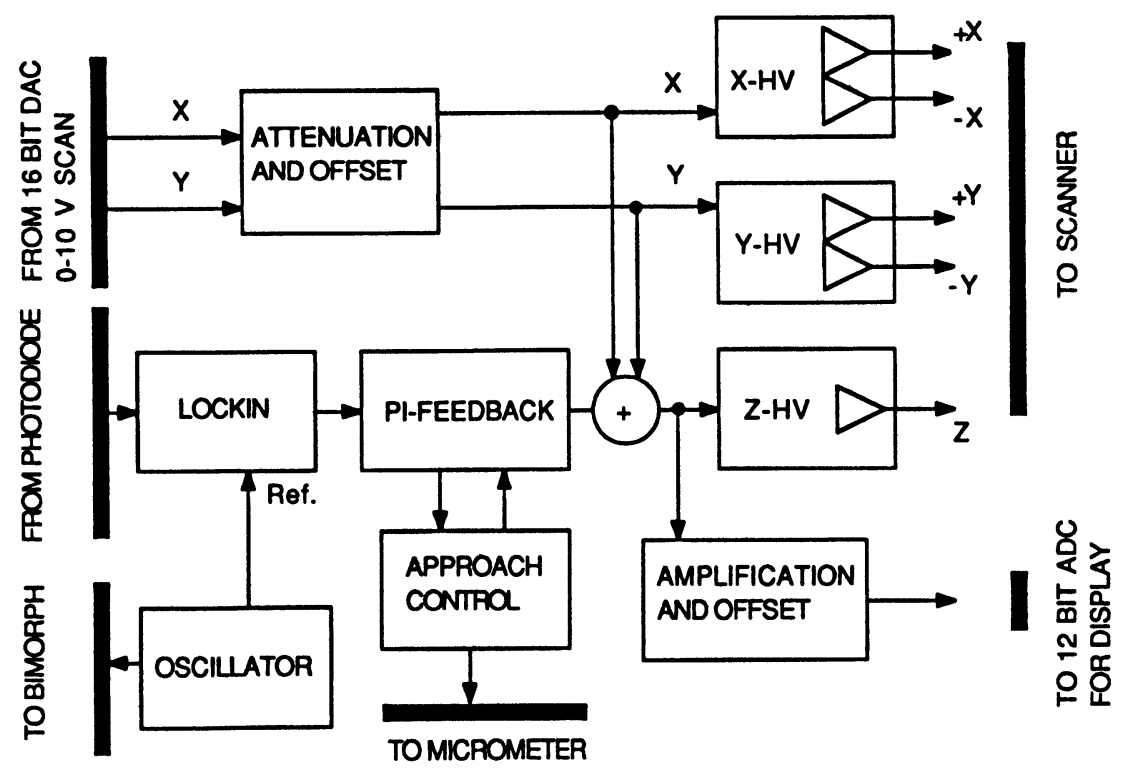

Fig. 4. - Schematic diagram of the control system.

It is, however, often possible to use the damped amplitude signal to regulate the $z$-voltage and to obtain images. The resonance detection method is discussed in more detail in the references $[5,15,21]$. 
In order to obtain an automatic approach sequence, a special circuit was designed that controls the coarse adjustment micrometer as well as the $z$-motion of the scanner. This circuit works as follows: Initially the scanner is fully expanded in the $z$-direction as the coarse approach is started with its lowest speed $(0.5 \mu \mathrm{m} / \mathrm{s})$. As the amplitude signal from the lock-in amplifier reaches a preset threshold corresponding to a weak interaction, the coarse approach is halted at the same time as the sample is retracted by switching the $z$-voltage on the scanner. At this stage, the tip is within reach of the scanner and the final approach can be started. This is done by applying a voltage ramp to the $z$-electrode of the piezo-tube, causing the sample to approach the tip with a controlled speed (125-850 $\AA / \mathrm{s}$ for the tube scanner). At contact, the circuit will either let the feedback control take over (if a measurement is started) or retract the sample. In the latter case, a sample and hold circuit will retain the voltage at which contact occurred and display the value for further reference.

As interface between the system and the computer we use an I/O board with four DAC:s (two 16 bit and two 12 bit) and eight ADC:s (12 bit) [22]. The 16 bit analog outputs generate the $x$ and $y$ scans, while one of the 12 bit outputs sweeps the oscillator frequency, an option that is used when acquiring frequency spectra of the lever resonance. The remaining 12 bit output controls the $z$-position of the sample, which is used when recording approach curves (see Sect. 3.2). The topographic signal which is fed into one of the ADC:s is derived from the $z$-voltage using an amplifier with stepwise variable gain and continuous offset. One of the analog inputs measures the cantilever amplitude (used when recording frequency spectra) and one monitors the lever displacement signal from the photodiode. The remaining ADC:s are used to monitor the sample bias voltage and different switch settings.

The fixed $0-10 \mathrm{~V}$ scan voltages from the computer are attenuated and mixed with a continuously variable offset before amplification in order to obtain the full resolution of the DAC:s, independent of the actual scan size. The $x$ and $y$ high voltage amplifiers are designed using two pairs of integrated circuits (Burr-Brown 3583) to obtain a symmetric voltage with respect to ground that is applied to the opposite electrodes of the tube scanner.

As a result of the high sensitivity to topographic variations, even a very small tilt of the sample with respect to the scan plane can lead to a severe distorsion of the image. This can be corrected for by adding a certain percentage of the $x$ and $y$ voltages to the $z$-voltage before it is amplified. In this way we obtain a slope correction in hardware that allows us to use the full resolution of the $\mathrm{ADC}$ for the topographic variations.

The attenuation and offset of the $x$ and $y$ voltages, as well as the amplification of the topography signal is read by the computer to provide all relevant information on the screen.

3.2 COMPUTER SOFTWARE. - The software is written in Lightspeed Pascal with additional low level commands from the "Macintosh Toolbox" [23] used for time critical data transfers to the screen. The program generates the scan voltages during data acquisition, displays the data and the relevant image parameters in real time, handles data storage and performs image processing. The computer is also used to record lever frequency spectra and to acquire approach curves, i.e. plots of static force (lever deflection) and force derivative (frequency shift) as a function of $z$-position while moving in and out of the interaction region $[15,24]$. These curves are not used during normal imaging but give the possibility to study the details of the tip-sample interaction.

The software allows images of up to $400 \times 400$ pixels and the graphics has a depth resolution of 256 levels. During data acquisition, the image is displayed as a collection of simple line scans where the topographic coordinate is added to the $y$-coordinate for each individual scan. Each scan direction will produce an image, so each complete acquisition will create two images that can be stored and treated individually.

Apart from the data acquisition and control functions, the program includes a comprehensive 
data storage and image processing package which allows the data to be displayed as line scans, intensity modulated top-views with different colour scales and 3-D views. The 3-D algorithm calculates a pixel intensity that is proportional to the scalar product between the normal vector of the surface element and a light vector "illuminating" the surface from an angle [25], while the pixel positions are determined by an algorithm giving a three dimensional projection of the pixel positions. Slope correction can be performed in software (as well as in hardware, see Sect. 3.1) by fitting a plane to the data points using regression analysis and subtracting the plane coordinates from the data points before display. The distribution of pixel colour values in the images can be displayed in histogram form. This function also gives the option to determine which topographic values that should define the extreme ends of the colour scale which is important as the automatic scaling otherwise would give a low contrast to an image with a few unrepresentative data points. A filtering function using convolution filtering is included as well as an interpolation function that allows an image with less than 200 data points in $x$ or $y$ to be presented as a $200 \times 200$ image which is the standard format. A multiview function allows the simultaneous display of four individual images on the screen for comparison.

Beside the normal binary file format containing the image data points, an additional file structure is available called "Multifiles", containing the names of up to six individual binary files. Accessing a file of this type will display six images stored on the hard disk without transfer of the binary data to the memory. By pointing at one of the displayed images with the cursor and clicking, the image is transferred to the memory and available for further viewing and processing. This option is helpful in organizing the data as images belonging together can be grouped, compared and accessed with a minimal delay.

\section{Results.}

As examples of instrument performance we show an image of an optical disk using a $2 \mu \mathrm{m}$ scan window, figure 5 , and a high resolution image of a fibrinogen molecule adsorbed on a silicon oxide substrate, figure 6.

The optical disk shown in figure 5 is a plastic replica covered with a $500 \AA$ metallic layer, and was imaged without bias voltage between tip and sample. The data is shown both as an intensity modulated top-view (a) and a 3-D rendered image (b) which clearly shows the visual impact of the latter image processing.

Figure 6 shows a top-view and a 3-D rendered image of a fibrinogen molecule deposited on a silicon oxide substrate that had been silanized to obtain a hydrophobic surface. The molecules were attached to the surface by immersion in a $0.2 \mu \mathrm{g} / \mathrm{ml}$ fibrinogen solution for 4 hours, followed by rinsing in distilled water and drying in clean nitrogen. The tungsten lever used has a resonance frequency of $21.9 \mathrm{kHz}$, and the size of the amplitude shift indicated that a capillary condensation phenomena contributed to the force interaction. The image shows clearly the tri-nodular structure of the fibrinogen molecule that is well known from TEM studies [26]. The lateral resolution in this image is estimated to $30 \AA$, and is obtained routinely. The data shown is a part of an ongoing study of fibrinogen interaction with solid surfaces that will be published separately. Molecular imaging of biological molecules using the SFM operated with repulsive forces has been reported elsewhere [27] but this is to our knowledge the first time that the shape of an individual bio-molecule has been observed when operating the SFM in the attractive force regime. 


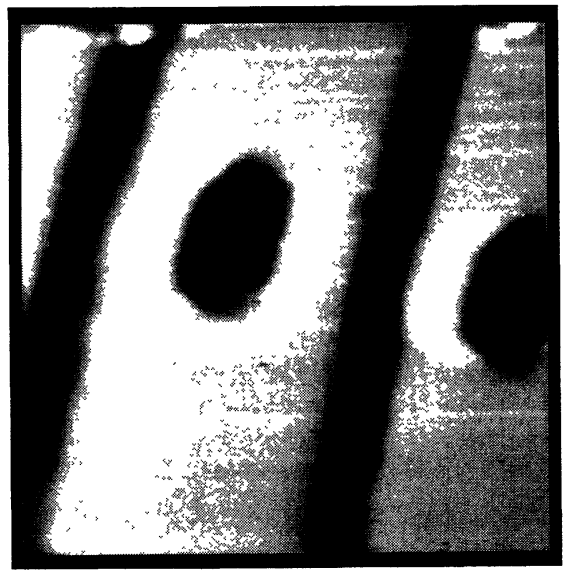

$2 \mu \mathrm{m}$

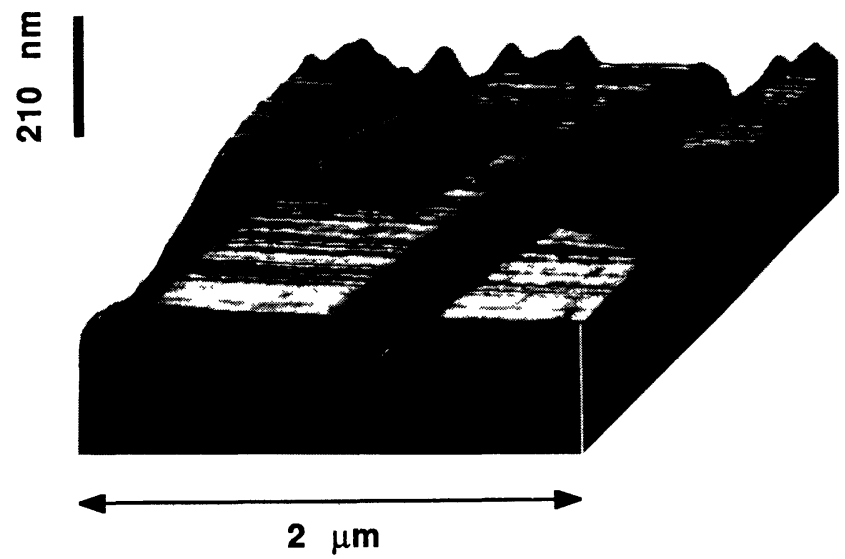

b)

Fig. 5. - (a) Top-view image of an optical disk. (b) 3-D rendered image showing the same data as in (a). See the text for further details.

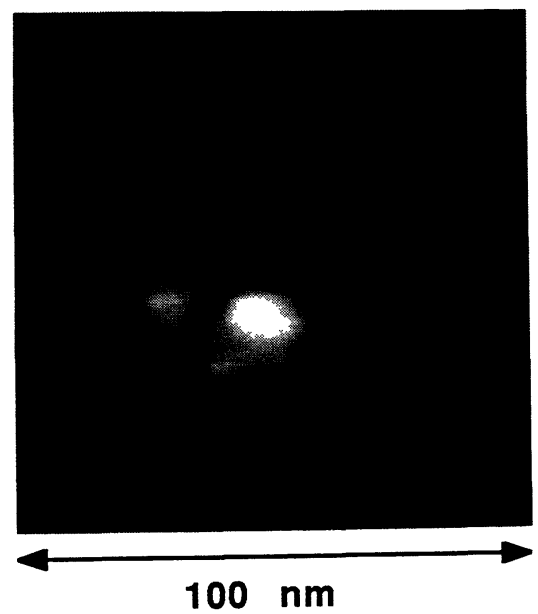

a)

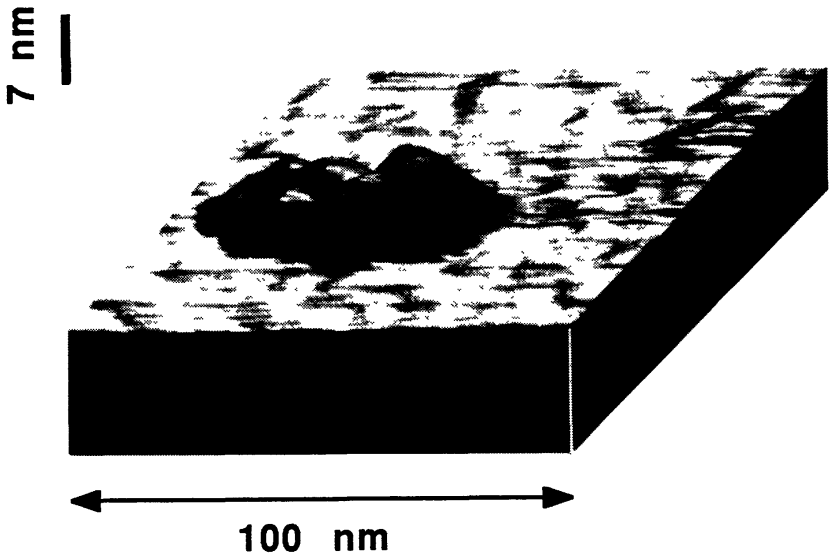

b)

Fig. 6. - (a) Top-view image of a fibrinogen molecule adsorbed on hydrophobic silicon oxide. (b) 3-D rendered image of a the same data as in (a). See the text for further details.

\section{Conclusions.}

We have presented a Scanning Force Microscope designed for general purpose use. The instrument includes a force sensor using an optical fiber interferometer, an interchangeable scanner system, control electronics and a comprehensive data acquisition and image processing software package. The images we present on fibrinogen adsorbed on hydrofobic silicon oxide show that 
the morphology of individual bio-molecules can be resolved on a routine basis with the scanning force microscope operated in the attractive regime.

\section{Acknowledgements.}

We thank Agneta Askendal for help with preparation of the biological molecules and Dr. Per Mårtensson for stimulating discussions.

\section{References}

[1] BinNig G., QUATE C.F. and Gerber Ch., Phys. Rev. Lett. 56 (1986) 930.

[2] BinNig G., GERBER Ch., STOLl E., AlBRECHT T.R. and QUATE C.F., Europhys. Lett. 3 (1987) 1281.

[3] Gould S.A.C., Drake B., Prater C.B., Weisenhorn A.L., Manne S., Hansma H.G., Hansma P.K., MAssie J., Longmire M., Elings V., Dixon NorThern B., MuKergee B., PeTERSON C.M., StOECKENIUS W., AlBRECHT T.R. and QUATE C.F., J. Vac. Sci. Technol A8 (1990) 369.

[4] RugaR D., MAMIN H.J., GUETHNER P., LAMBERT S.E., STERn J.E., McFADYEN I. and YOGI T., J. Appl. Phys. 68 (1990) 1169.

[5] Martin Y., Williams C.C. and WiCKRamasinghe H.K., J. Appl. Phys. 61 (1987) 4723.

[6] Mate C.M., McClelland G.M., ERlandsson R. and Chiang S., Phys. Rev. Lett. 59 (1987) 1942.

[7] Marti O., DraKe B., Gould S. and HaNSMa P.K., J. Vac. Sci. Technol. A6 (1988) 287.

[8] KIRK M.D., ABRECHT T.R. and QUATE C.F., Rev. Sci. Intrum. 59 (1988) 833.

[9] BUUgler D., Heinzelmann H., TARRACH G., MEYER E. and GunTERHEROdT H.-J., Presented at the STM 90 Fifth international Conference on Tunneling Microscopy/Spectroscopy, Baltimore (July 1990).

[10] Cohen S.R., Neubauer G., McClelland G.M., Coulman D. and Brown C.A., Presented at the STM 90 Fifth international Conference on Tunneling Microcopy/Spectroscopy, Baltimore (July 1990).

[11] Rugar D., Mamin H.J. and GueThNer P., Appl. Phys. Lett. 55 (1989) 2588.

[12] LEFEVRE H.C., Electron. Lett 16 (1980) 778.

[13] Rugar D., MAMin H.J., ERLANDSSON R., Stern J.E. and TerRis B.D., Rev. Sci. Instrum. 59 (1988) 2337.

[14] Heinzelmann H., Meyer E., GRUTter P., Hidber H.-R., ROSENTHALER L. and GUNTHEROdT H.-J., J. Vac. Sci. Technol A6 (1988) 275.

[15] ERlandsson R., McClelland G.M., Mate C.M. and Chiang S., J. Vac. Sci. Technol. A6 (1988) 266.

[16] FASTH J.E., LOBERG B. and NORDÉN H., J. Sci. Instrum. 44 (1967) 1044.

[17] BINNIG G. and SMITH D.P.E., Rev. Sci. Instrum. 57 (1986) 1688.

[18] Staveley Sensor Inc. East Hartford, CT 06108, U.S.A.

[19] CARR R.G., J. Microsc. 152 (1988) 379.

[20] P-280 Block Translator, Physik Instrumente (PI) GmbH, Siemenstrasse, D-7517 Waldbronn, Germany.

[21] Nonnenmacher M., PhD Thesis, Rastermikroskopie mit Mikrospitzen, Fachbereich Physik der Gesamthochschule Kassel Universităt des Landes Hessen (1990).

[22] GW Instruments, Inc, P.O. Box 2145, Cambridge, MA 02141, U.S.A.

[23] Inside Macintosh, vol I, II and III (Addison-Wesley Publishing Company, Inc).

[24] Burnham N.A. and COlTON R.J., J. Vac. Sci. Technol. A7 (1989) 2906.

[25] FisHMAN B. and SCHACHTER B., Comput Graphics 5 (1980) 53.

[26] NYGREN H. and STENBERG M., J. Biomed. Mater. Res. 22 (1988) 1.

[27] DRAKe B., Prater C.B., WeISENHORN A.L., GOULD S.A.C., AlBRECHT S.T.R., QuATE C.F., CANELlI D.S., HANSMA H.G. and HANSMA P.K., Science 243 (1989) 1586. 